\title{
Refocusing the Vietnam HIV surveillance to the most burden areas for epidemic control
}

\author{
Diep T. Vu¹, Duc H. Bui ${ }^{2}$, Giang T. Le ${ }^{1}$, Duong C. Thanh ${ }^{3}$, Nghia V. Khuu ${ }^{4}$, \\ Abu Abdul-Quader ${ }^{1}$ and Huong T. Phan ${ }^{2}$
}

${ }^{1}$ Centers for Disease Prevention and Control, Hanoi, Viet Nam; ${ }^{2}$ Vietnam Authority of HIV/AIDS Control, Hanoi, Viet Nam; ${ }^{3}$ National Institute of Hygiene and Epidemiology, Hanoi, Viet Nam; ${ }^{4}$ Pasteur Institute in Ho Chi Minh City, Hanoi, Viet Nam

\section{Objective}

To describe an exercise to identify priority provinces to be focused in the Vietnam National HIV Sentinel Surveillance (HSS).

\section{Introduction}

The Vietnam National HSS was established in 1994. In the late 1990 s and early 2000s, when the epidemic was increasing rapidly, the HSS helped with the intensive close monitoring of the HIV epidemic. In its first 10 years, the HSS was rapidly expanded from 6 to 40 provinces and in some years, it was conducted semi-annually. After two decades, the HIV epidemic situation has changed. In most provinces, HIV prevalence has reported to have declined. Compared to the peak period, the HIV prevalence among key populations (KP) in the past decade decreased from $40-60 \%$ to $20 \%$ or lower. In many provinces, HIV prevalence was less than $10 \%$ among people who inject drugs (PWID) and less than 3\% among female sex workers (FSW), and among men who have sex with men (MSM) (Table 1). At the same time, the HIV programme has since been scaled up widely with various interventions and expanded to most of the 63 provinces. In 2014, the government of Vietnam and international stakeholders conducted a joint review of the health sector response to the HIV epidemic and concluded that for better monitoring of the epidemic, a more focused and higher quality surveillance system was needed ${ }^{(1)}$. In 2015, surveillance stakeholders conducted a detailed review of the HSS to discuss prioritization of the surveillance activities.

\section{Methods}

The prioritization exercise followed a principle that the HSS should be conducted in locations where there is a large population of KP with a high HIV prevalence and it is feasible to implement. Criteria for prioritizing provinces for inclusion were: 1) a high estimated KP size; 2) high HIV prevalence, measured as a 5 year (2011-2015) average prevalence (P); 3 ) few years with low HIV prevalence, defined as $\mathrm{P}$ $<5 \%$ among PWID, $<3 \%$ among FSW and MSM; 4) few years with insufficient HSS sample size, defined as $\mathrm{n}<150$ for PWID, $\mathrm{n}<250$ for FSW and MSM. Steps to prioritize provinces were:

- Reviewed provincial data on KP estimates; HIV prevalence and achieved HSS sample sizes in 5 years, 2011-2015.

- Developed a ranking algorithm taking into account KP size estimates, HIV prevalence and achieved sample sizes.

- For each survey on PWID, FSW, MSM, took top ranked provinces for which sum of KP size estimates of these provinces exceeded $50 \%$ of the national KP size estimates.

- Held a consultation workshop among domestic and international surveillance stakeholders to discuss the prioritization exercise. Issues of regional representation of the HSS in the North, South, Central and Highland regions was added as a criteria to adjust the priority list of HSS provinces. The consensus reached in the workshop was the basis for proceeding a formal approval at Ministry of Health.

\section{Results}

The data review and panel discussion suggested that the number of provinces to implement HSS should be 20 for PWID, 13 for FSW, and
7 for MSM surveys. While total number of provinces reduced from 40 to 20, all 4 geographical regions of the country were covered. Even with the reduction of the geographical coverage of the HSS, large proportions of the KPs $(63.9 \%$ of PWID, $58.9 \%$ of FSWs and $36 \%$ of MSM) were covered under the HSS (Table 2). In February 2017, the Ministry of Health officially approved the 20 priority provinces as a part of the new strategic direction of the Vietnam National HSS.

\section{Conclusions}

Adjusting the HSS to better align it with the HIV epidemic and programmatic needs is necessary. Refocusing the HSS to high burden epidemic areas saves time and resources, thus enables more focus on data quality improvement. Innovation to advance survey methods, adherence to survey protocol, and additional bio-markers to better monitor the epidemic will be the emphasis of the HSS towards a more timely and robust surveillance system in Vietnam.

Table 1: Status of the HSS before the prioritization exercise

\begin{tabular}{|c|c|c|c|}
\hline & PWID & FSW & MSM \\
\hline No. HSS province & 38 & 40 & 13 \\
\hline HIV prevalence (P)* & & & \\
\hline - Mean & $12.6 \%$, & $3.0 \%$, & $2.6 \%$, \\
- Median & $11.9 \%$, & $2.1 \%$, & $1.4 \%$, \\
- Min-Max & $1.7 \%-$ & $0.0 \% \%-$ & $0.4 \%-$ \\
\hline No. and \% of HSS province with & $33.8 \%$ & $20.5 \%$ & $12.0 \%$ \\
\hline$-\mathrm{P}<10 \%$ & & & \\
\hline$-\mathrm{P}<5 \%$ & $22(57.9 \%)$ & $39(97.5 \%)$ & $12(92.3 \%)$ \\
\hline$-\mathrm{P}<3 \%$ & $10(26.3 \%)$ & $35(87.5 \%)$ & $11(84.6 \%)$ \\
\hline Needed sample size per survey & $2(5.3 \%)$ & $30(75.0 \%)$ & $8(61.5 \%)$ \\
\hline No. and \% of HSS province with 3 or more years of not achieving sufficient & $150-300$ & $300-500$ & $300-500$ \\
\hline sample size*** & $1(1.7 \%)$ & $24(60.0 \%)$ & $5(35.7 \%)$ \\
\hline
\end{tabular}

* Five year (2011-2015) average HIV prevalence

** $\mathrm{N}<150$ for PWID survey and $\mathrm{N}<250$ for FSW or MSM survey

Source: National HIV sentinel surveillance database, Ministry of Health.

Table 2: Geographical and population coverage of the HSS before and after the prioritization.

\begin{tabular}{|c|c|c|c|c|}
\hline & HSS coverage & Before 2017 & After 2017 & Change \\
\hline PWID & No. provinces & 37 & 20 & 17 \\
\hline & KP size estimates & 227,038 & 174,505 & 52,533 \\
\hline & $\%(*)$ & $83.20 \%$ & $63.90 \%$ & $-19.20 \%$ \\
\hline FSW & No. provinces & 40 & 13 & 27 \\
\hline & KP size estimates & 59,994 & 42,740 & 17,254 \\
\hline & $\%(*)$ & $82.60 \%$ & $58.90 \%$ & $-23.80 \%$ \\
\hline MSM & No. provinces & 14 & 7 & 7 \\
\hline & KP size estimates & 179,931 & 139,702 & 40,229 \\
\hline & $\%(*)$ & $46.80 \%$ & $36.30 \%$ & $-10.50 \%$ \\
\hline
\end{tabular}

* \% compared to national KP size estimates

\section{Keywords}

HIV/AIDS; sentinel surveillance; policy 


\section{Acknowledgments}

VAAC, Regional Institutes and Provincial HIV/AIDS Centers for their dedicate work on the HSS. The technical assistance to strengthen the HSS has been supported by the President's Emergency Plan for AIDS Relief (PEPFAR) through the CDC.

\section{References}

1. World Health Organization. Regional Office for the Western Pacific, 2016, Joint Review of the Health Sector Response to HIV in Viet Nam 2014.

\section{*Diep T. Vu}

E-mail:vubichdiep@hotmail.com 